\title{
An Environmental Dilemma for China During the COVID-19 Pandemic: The Explosion of Disposable Plastic Wastes
}

\author{
Jianli Liu' ${ }^{1}$ - A. Dick Vethaak ${ }^{2,3} \cdot$ Lihui An $^{4} \cdot$ Qiang Liu $^{5} \cdot$ Yunfei Yang $^{6} \cdot$ Jiannan Ding $^{7}$ \\ Received: 12 December 2020 / Accepted: 18 January 2021 / Published online: 29 January 2021 \\ (C) The Author(s), under exclusive licence to Springer Science+Business Media, LLC part of Springer Nature 2021
}

\begin{abstract}
Plastic pollution control has been on top of the political agenda in China. In January 2020, China announced a phased ban on the production and usage of various types of single-use plastics as a solution to environmental pollution problems. However, the outbreak of COVID-19 seems to be a new obstacle to the ban on single-use plastic products. To basically satisfied the daily necessities and contain the spread of SARS-CoV-2 under the background of the regular epidemic prevention and control in China, online ordering, contactless delivery and wearing mask have become an important and feasible way of daily life. However, the unrestrained use of disposable plastic bags, lunch boxes and masks within the nationwide quarantine leads to hundreds of millions of plastics wastes every day. The potential environmental pollution caused by the use of disposable plastic products during the pandemic should arouse social concern. The Chinese government should manage environmental protection in parallel with anti-pandemic endeavors as the situation of the pandemic evolves.
\end{abstract}

Keywords Environmental dilemma $\cdot$ COVID-19 pandemic $\cdot$ Disposable plastic packaging $\cdot$ Online shopping $\cdot$ Contactless delivery $\cdot$ Plastic pollution

Plastic pollution control has been on top of the political agenda in China. In January 2020, China announced a phased ban on the production and usage of various types of single-use plastics as a solution to environmental pollution problems. The proposal involved banning plastic bags in all major cities by the end of 2020 and nationwide by 2022 . However, the COVID-19 outbreak emerged as an obstacle against this new initiative. To prevent crowding and prevent the spread of the virus, online shopping and contactless

Jianli Liu

Jian-li.liu@ @otmail.com

A. Dick Vethaak

Dick.Vethaak@deltares.nl

Lihui An

anlhui@163.com

Qiang Liu

Liu.qiang@h.mbox.nagoya-u.ac.jp

Yunfei Yang

3677986@qq.com

Jiannan Ding

djn@jiangnan.edu.cn

1 School of Textile Science and Engineering, Jiangnan University, Wuxi 214021, China delivery have become the new modes for the purchase of essentials. These new modes have also assured the survival of the catering and retailing industries, which have suffered heavy losses because of the shutdown of nearly all restaurants, supermarkets, and stores during the early period of the pandemic (Vasiev et al. 2020). In this situation, plastic packaging is now a necessity for takeout and express delivery. Single-use plastic packaging is widely used because of concerns about the spread of COVID-19 through reusable

2 Deltares, Marine and Coastal Systems, Boussinesqweg 1, Delft 2629 HV, the Netherlands

3 Department of Environment and Health, Vrije Universiteit Amsterdam, De Boelelaan 1085, Amsterdam 1081 HV, the Netherlands

4 State Key Laboratory of Environmental Criteria and Risk Assessment, Chinese Research Academy of Environmental Sciences, Beijing 100012, China

5 Bioscience and Biotechnology Center, Nagoya University, Nagoya 4648601, Japan

6 Jiangsu Provincial Academy of Environmental Science, Nanjing 210036, China

7 School of Environmental and Civil Engineering, Jiangnan University, Wuxi 214122, China 
items (Parashar and Hait 2021). Furthermore, coupled with the sharp drop in global crude oil prices, the cost of raw materials for plastics production will become even cheaper (Gharib et al. 2020). These factors may emerge as potential driving forces to further promote the mass usage of plastic products in e-commerce, catering, and express delivery, reversing the trend of the plastic ban.

As a crucial step towards stemming the spread of the virus, the Chinese government decisively enforced a community quarantine across the nation from Jan. 23 to Feb. 21, 2020 (Kraemer et al. 2020). A 76-day lockdown and community quarantine were especially enforced in Wuhan, the epicenter of the pandemic to contain the outbreak. Despite strict prevention and control measures such as traffic control and home quarantine which are inconvenient and have impeded the movement of people, online ordering and contactless delivery maintained the supply of daily necessities in urban areas. Making each community a unit, residents undertook centralized online ordering of daily necessities. Couriers brought products into the community and community workers and volunteers delivered them to residents through doorstep delivery. Data released by the Wuhan Municipal Bureau of Commerce on Feb. 10, 2020, showed 15 large- and medium-sized e-commerce platforms in the city that conducted the distribution of fresh food online, with an average daily delivery volume of more than 160,000 orders (Wuhan Municipal Bureau of Commerce 2020). It is conservatively estimated that more than 1.216 million centralized online orders were delivered in Wuhan city before the reopening on April 8, 2020. For example, each family purchased four types of vegetables every time. Each type was individually packaged and placed in a large plastic bag marked with the purchaser's information. This means that at least five plastic bags were used for each order. Optimistically, at least 6.08 million disposable plastic bags were used for packaging in the lockdown period. If the average area of each plastic bag was 0.06 square meters (the actual size of plastic bags used in supermarkets and vegetable markets is much larger), the total area of plastic packaging used in Wuhan city would exceed 3.648 million square meters, an area large enough to cover more than 51 football fields. This does not include disposable plastic materials for other uses, such as lunch boxes and tableware. Mainland China has 19 first-tier cities like Wuhan and 337 prefecture-level cities (Zheng et al. 2018). It is estimated that, in only the 19 firsttier cities, more than 500 million disposable plastic bags were used within the 30-day lockdown period. The COVID19 data released by the China Council's Joint Prevention and Control Mechanism indicated that more than 130,000 orders of cooked food were delivered through online ordering and courier delivery on Feb. 25, 2020 (Xinhuanet 2020). On the same day, the catering industry's resumption rate reached $57 \%$, based on the investment of 20 large-scale chain catering companies in Wuhan city (Xinhuanet 2020). It is optimistically estimated that only one lunch box was used for each order. If the height of a lunch box was $5 \mathrm{~cm}$, the cumulative height of lunch boxes used in Wuhan city in the period between Feb. 25 and April 8 would be equivalent to 31 time the height of Mount Everest. Undoubtedly, because of online shopping, hundreds of millions of pieces of plastic garbage were generated during the nationwide lockdown. The explosion of disposable plastic packaging during the COVID-19 pandemic is visualy described in Fig. 1. On top of this, the crucial need for personal protective equipment also increased the usage of plastic-based fibrous materials (Prata et al. 2020). The daily output of medical N95 masks and medical non-N95 masks increased from 130,000 and 5.86 million in early February to over 5 million and 200 million by the end of April (SCIO 2020). Although the nonrecyclable disposable plastic waste is landfilled and incinerated, there is a potential environmental risk of plastic losses, the release of microfibers, and toxic chemicals (Rochman and Hoellein 2020).

The COVID-19 pandemic has greatly increased our reliance on plastics to provide safety protection and daily supplies. However, single-use plastic packaging and personal protective equipment (PPEs) are potential sources of pollution for the land and aquatic environment. The temporary and explosive growth of disposable plastic packaging will sharply increase waste volume, which has been a great challenge for the conventional facilities of municipal solid wastes. The mismanagement and leakage of disposable plastic packaging aggravate issues of plastic pollution, pose an existential threat to soil, air, and aquatic ecosystems, and cause potential damage to wildlife and humans health (Barboza et al. 2018). Single-use PPEs, such as masks, gloves, disinfectant wipes, protective suits, and other coronavirus wastes have been found in the environment. The increasing amounts of single-use PPEs and health care wastes (HCWs) caused by COVID-19, a new form of pollutant, recently have concerned the international community (Vanapalli et al. 2021). Compared with disposable plastic packaging, PPEs and HCWs have higher security and environmental risk due to the high infectiousness and strong survivability of the SARS-CoV-2 virus (Yang et al. 2021). PPEs and HCWs are made of synthetic fibrous materials with a lightweight structure, low density, and a large surface area, and thus spread more easily in the environment.

In 2020, COVID-19 and environmental pollution have put a double burden on the Chinese government. The COVID19 pandemic, a historic health emergency, and a daunting challenge may have temporarily and severely interrupted the plastic ban. However, an active response to COVID19 and plastic pollution is urgently needed to ensure not only people's health and safety but also ecological security. Nowadays, consumer behavior and shopping pattern have 


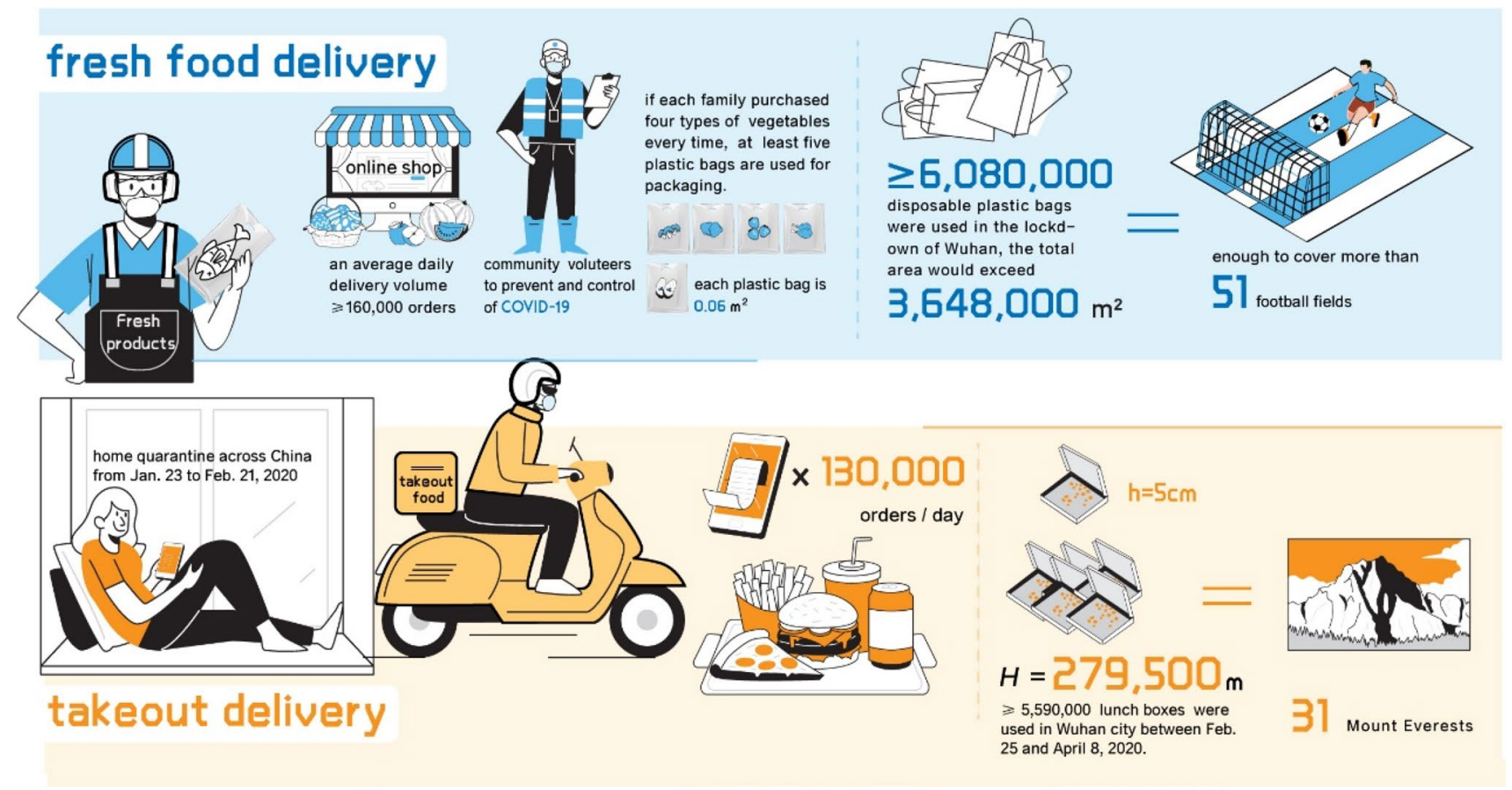

Fig. 1 The explosion of disposable plastic packaging during the COVID-19 pandemic

been forcibly changed by the COVID-19 outbreak. Online ordering and contactless delivery have gradually formalized as normal consumer behavior, and therefore more plastic products will be passively or actively used by urban and rural residents. The potential environmental pollution caused by the use of disposable plastic products during the pandemic should arouse social concern. The health and security concerns demand that each government in the world should mandatorily manage environmental protection in parallel with anti-pandemic endeavors.

The emergency response to and the security management of the explosive growth of disposable plastic packaging during the COVID-19 pandemic are obligatory for every government. Firstly, emergency investment should be made in infrastructure, and more professional sanitation workers should be recruited to increase the treatment capacity. The consecutive utilization of mobile facilities and the expansion of daily treatment capacity of stationary facilities, incineration plants, and nonlocal disposal were effective approaches to deal with the explosive growth of health care wastes during the COVID-19 pandemic in Wuhan, China (Yang et al. 2021). Secondly, these plastic packaging, PPEs, and HCWs should always be disposed of responsibly to contain the spread of SARS-CoV-2 and reduce the negative impact on the environment. The medical waste management regulations should be referenced and applied to the segregation, collection, storage, transportation, and disposal stage of plastic wastes during the COVID-19 pandemic. Now, it's necessary to make a universal action plan, rather than some provisional acts and temporary policies. Thirdly, much greater regulatory incentives for people to recycle and reuse are needed. Individuals are encouraged to use reusable packaging and wear reusable masks after disinfection using a 75\% alcohol solution and UV lamps (Yang et al. 2021). If individuals wear reusable masks, not single-use masks, this will preserve single-use mask supplies for front-line healthcare workers, and reduce the risks associated with the disposal of contaminated plastic mask (UCL Plastic Waste Innovation Hub 2020). As pandemic evolves, the governments of China and other countries must coordinate their epidemic control measures with those of environmental protection to achieve an optimal trade-off.

Acknowledgements The authors would like to express our gratitude to all aid workers and medical personnel fighting at the frontline against COVID-19.

Author contributions JLL conceived the study, collected the data and drafted the manuscript; ADV provided critical feedback and helped shape the analysis and manuscript. LHA contributed to the design of the study and carried out data analysis and plotted the figure; QG, YFY and JND coordinated the study tasks, and helped draft the manuscript. All authors gave final approval for publication.

Funding This study was supported by the National Natural Science Foundation of China (No. 51809118), the Natural Science Foundation of Jiangsu Province, China (No. BK20170188).

Data Availability Not applicable. 


\section{Compliance with Ethical Standards}

Conflict of Interest The authors declare that they have no competing interests.

Ethical Approval Not applicable.

Consent to Participate Not applicable.

Consent for Publication Not applicable.

\section{References}

Barboza LGA, Dick Vethaak A, Lavorante BRBO et al (2018) Marine microplastic debris: an emerging issue for food security, food safety and human health. Mar Pollut Bull 133:336-348. https:// doi.org/10.1016/j.marpolbul.2018.05.047

Gharib C, Mefteh-Wali S, Ben JS (2020) The bubble contagion effect of COVID-19 outbreak: evidence from crude oil and gold markets. Financ Res Lett. https://doi.org/10.1016/j.frl.2020.101703

Kraemer MUG, Yang CH, Gutierrez B et al (2020) The effect of human mobility and control measures on the COVID-19 epidemic in China. Science 368:493-497. https://doi.org/10.1126/ science.abb4218

Parashar N, Hait S (2021) Plastics in the time of COVID-19 pandemic: protector or polluter? Sci Total Environ 759:1-16

Prata JC, Silva ALP, Walker TR et al (2020) COVID-19 pandemic repercussions on the use and management of plastics. Environ Sci Technol 54:7760-7765. https://doi.org/10.1021/acs.est.0c02178
Rochman CM, Hoellein T (2020) The global odyssey of plastic pollution. Science 368:1184-1185. https://doi.org/10.1126/scien ce.abc 4428

SCIO (2020) Fighting COVID-19: China in Action

UCL Plastic Waste Innovation Hub (2020) The environmental dangers of employing single-use face masks as part of a COVID-19 exit strategy

Vanapalli KR, Sharma HB, Ranjan VP et al (2021) Challenges and strategies for effective plastic waste management during and post COVID-19 pandemic. Sci Total Environ 750:141514. https://doi. org/10.1016/j.scitotenv.2020.141514

Vasiev M, Bi K, Denisov A, Bocharnikov V (2020) How COVID-19 pandemics influences chinese economic sustainability. Foresight Sti Gov 14:7-22. https://doi.org/10.17323/2500-2597.2020.2.7.22

Wuhan Municipal Bureau of Commerce (2020) Promote e-commerce distribution, solve shopping problems, and reduce gathering risks(in Chinese). In: Wuhan Munic. Bur. Commer. http:// sw.wuhan.gov.cn/xwdt/mtbd/202004/t20200423_1077542.shtml . Accessed 10 Feb 2020

Xinhuanet (2020) The number of food and beverage delivery in Wuhan reached 130,000 orders yesterday (in Chinese). In: www.Xinhu anet.com. http://www.xinhuanet.com/2020-02/26/c_1125629700 .htm. Accessed 26 Feb 2020

Yang L, Yu X, Wu X et al (2021) Emergency response to the explosive growth of health care wastes during COVID-19 pandemic in Wuhan. China Resour Conserv Recycl 164:105074. https://doi. org/10.1016/j.resconrec.2020.105074

Zheng B, Zhang Q, Davis SJ et al (2018) infrastructure shapes differences in the carbon intensities of chinese cities. Environ Sci Technol 52:6032-6041. https://doi.org/10.1021/acs.est.7b05654 